\title{
Fusi In Situ Posterior for Congenital Scoliosis with Wedge Hemivertebrae Using Single Rod and Pedicle Screw Instrumentation in Girl 2 Years Old: A Case Report
}

\author{
Pamudji Utomo, Adi Surya Dharma, Rieva Ermawan
}

\author{
Department of Orthopaedic \& Traumatology, Faculty of Medicine, \\ Universitas Sebelas Maret/ Prof. Dr. R. Soeharso Orthopaedic Hospital, Surakarta
}

\section{ABSTRACT}

Background: Many spinal vertebral anomalies often occur, both scoliosis and kyfosis. In scoliosis, congenital abnormalities caused by hemivertebrae are the most common cause of abnormalities. The choice of treatment used can be either non-operative or operative. However, non-operative treatment does not show satisfactory results to prevent the development of deformity. Operative treatment is an option that is considered to indicate more satisfying results. A variety of procedures such as in-situ posterior or with or without instrumentation anteroposterior fusions, combined anterior and posterior convex hemiepiphysiodesis and hemiarthrodesis, and hemivertebral excision with fusion. Fusi In-situ posterior is a procedure option that is considered more beneficial for both surgeons and patients. This study aimed to evaluate the fusi in situ posterior for congenital scoliosis with wedge hemivertebrae using single rod and pedicle screw instrumentation in girl 2 year old.

Case Presentation: Girl 2 year old with wedge hemivertebrae operated with fusion in situ posterior using single rod and pedicle screw instrumentation.

Results: The operative treatment was performed on 2 hours operation time with amount of bleeding produced is 80 cc. There were no cranckshaft phenomena and no clinical and radological features suggestive of spinal stenosis. There were no major vascular or neurogical complications related to the pedicle screws. Then patient wore body jacket for limitation movement before fusion.

Conclusion: Posterior situ fusion is performed as convex fusion to avoid curve progression. Fusion in situ posterior using single rod and pedicle screw instrumentation. In congenital scoliosis is minimal blood loss, less traumatic, simple, safe and effective procedure. This study showed the early fusi in situ posterior of congenital scoliosis structural changes occur above or below can reduce fusion length, prevent curve progression and effectively achieve a more satisfactory correction without hazardous iatrogenic spinal stenosis, crankshaft phenomena, or neurological complications. Further research is needed to assess the progress of the outcome of surgery

Keywords: congenital scoliosis, wedge hemivertebrae, convex, fusi in situ posterior

\section{Correspondence:}

Pamudji Utomo. Department of Orthopaedic \& Traumatology, Faculty of Medicine, Universitas Sebelas Maret/ Prof. Dr. R. Soeharso Orthopaedic Hospital, Surakarta

Cite this as:

Utomo P, Dharma AS, Ermawan R (2020). Fusi In Situ Posterior for Congenital Scoliosis with Wedge Hemivertebrae Using Single Rod and Pedicle Screw Instrumentation in Girl 2 Years Old: A Case Report. Indones J Med. 05(02): 125-130. https://doi.org/10.26911/theijmed.2020.05.02.05.

cc) (1) (2) Indonesian Journal of Medicine is licensed under a Creative Commons

\section{BACKGROUND}

Congenital scoliosis is a vertebral lateral curve disorder that causes an imbalance in longitudinal spinal growth. This abnormal- lity usually occurs in the first six weeks of intrauterine life, a pattern of anatomical compilation of bone shapes from mesenchymal tissue. Usually abnormalities have 
emerged since birth, but began to complain when growing up (Guren, 2013). The prevalence of worldwide incident of vertebral abnormalities is $0.5^{-1}$ per 1,000 live births (Debnath, 2010).

Hemivertebras are a common congenital scoliosis disorder. With several types of incarceration, for wedge-shaped deformities that develop during further spinal growth. Nonsurgical treatments, including bracing, have been frequently unsuccessful in preventing the progression of the deformity, and surgical intervention is required for most cases with curve progression. Operative therapy is an option that is considered to indicate more satisfying results. In-situ variety of procedures such as pos-

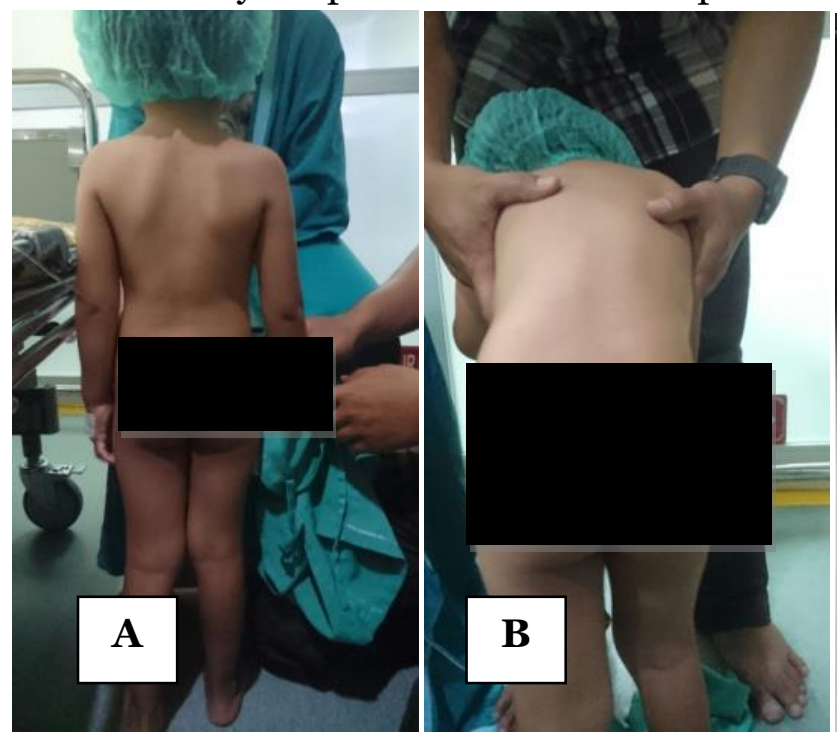

Figure 1 (A-B). Physical examination

On physical examination the patient can walk normally, in general condition, there is no abnormality. Examination of motor and sensoric neurological status has no weakness or abnormalities. Physiological reflexes are found normally in all four extremities. Whereas pathological reflexes were found by Hoffman (-/-), Tromner (-/), Babinski (-/-), Chaddox (-/-), Oppenheim terior or without instrumentation anteroposterior fusions, combined anterior and posterior convex hemiepiphysiodesis and hemiarthrodesis, and hemivertebral excision with fusion (Peng, 2011).

\section{CASE PRESENTATION}

The patient is a 2 years old girl who lives in Central Java. Her parent realized that her back bent when the age 1 year old. The curve increases progressively on 1 year evaluation. There was no complaintsof pain and dypsneau were found. no history of trauma, no fever, no weight loss, normal activity, normal appetite. The patient does not have a history of the same disease in his family.

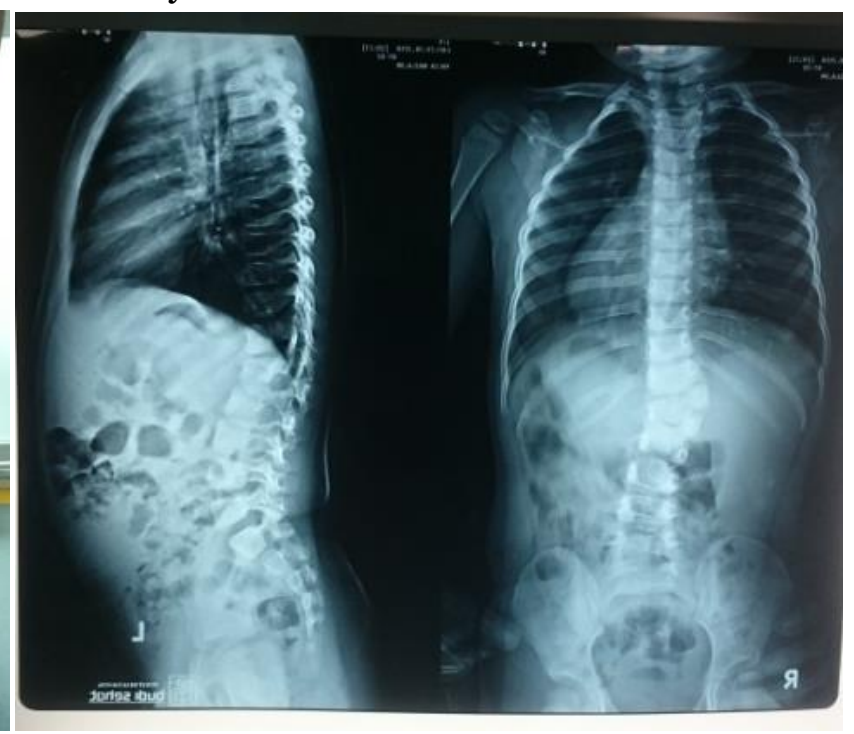

Figure 2. Pre operative $X$ ray

(-/-), and clonus (/-). Autonomic function is still within normal limits, there is no evidence of micturition and defecation disorder in patients.

On CT-Scan examination, there is an Wedge Hemivertebra in Vertebrae Thoracal 12(Figure 2). The structure of the vertebral body is still normal. 


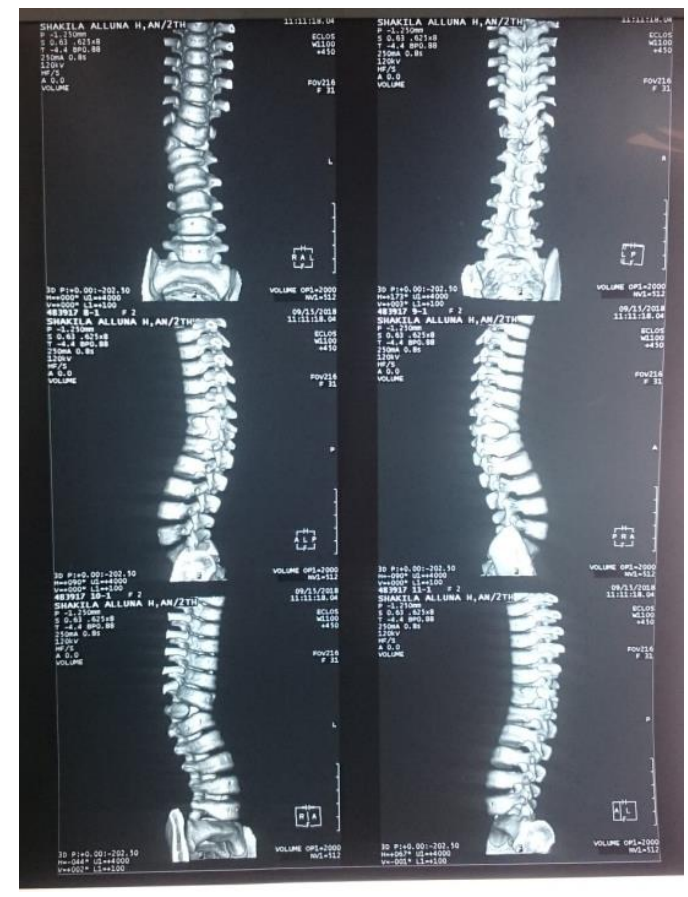

Figure 3. Pre Operatif CT-Scan

The patient was positioned prone on the bed and all pressure was released from the abdomen. A longitudinal paracentral incision from Vth-11 to VL-1was made from one segment cephalad to one segment caudal to the hemivertebra. The posterior aspects of the hemivertebra and the adjacent vertebrae in the convexity exposed were later to the tip of the transverse processes, and the posterior elements of the hemivertebra were identified. Hemivertebra is found at the Vth-12 Dextra. After that, a facetectomy was performed on Vth11 - VL1 in right side. attach the pedicle screw in Vth-11 and VL-1right side. Fusi in situ in dextra side and insert an allograft on the same side. wash the operating field, close it layer by layer and the operation is complete. Then patient is worn a body jacket with window on wound site.

\section{DISCUSSION}

The treatment goal for congenital scoliosis due to a hemivertebra is to achieve a straight spine with a physiological sagittal profile and as short a fusion segment as

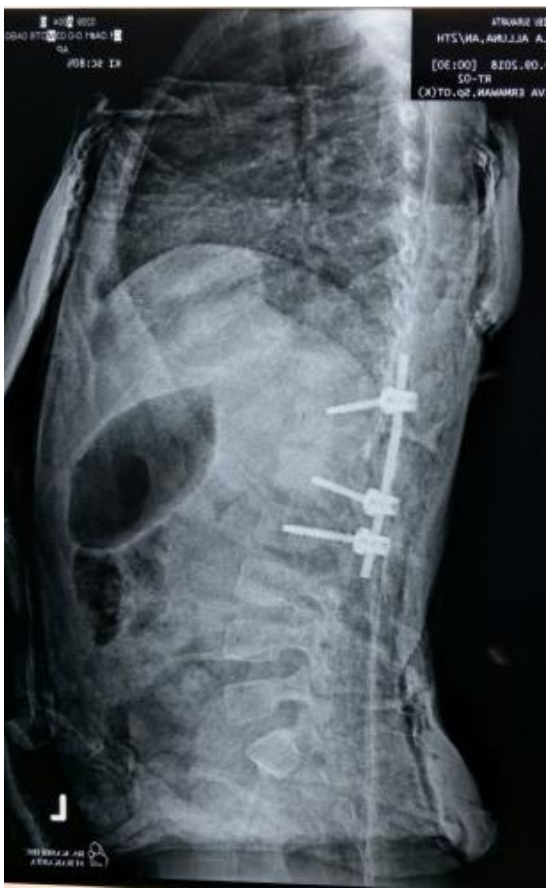

Figure 4. Post operative X-Ray

possible. Delayed treatment of an advanced deformity in older children or adults has to include the secondary structural curves and requires a more extensive correction procedure. For lower lumbar hemivertebrae in particular, the operation should be performed at an early stage before the secondary compensatory curve in the lumbosacral junction becomes rigid; otherwise, when the secondary compensatory curve in the lumbosacral region becomes rigid, trunk imbalance may occur after resectionof low lumbar hemivertebra and correction of scoliosis. Therefore, the key to successful treatment is early diagnosis and treatment. Excision of a hemivertebra not only removes the cause of deformity but also allows for superior and immediate deformity correction. Therefore, it is an ideal option for treatment of congenital scoliosis due to a hemivertebra (Nakamura, 2002).

The surgical descriptions of hemivertebra excision have focused on anterior and posterior exposures, using either a onestage or a two-stage procedure to complete the hemivertebra resection. The combined 
anterior and posterior approach allows the surgeon maximum visualization and control of the spine during hemivertebra excision. The anatomy can be reliably determined during surgery, and complete resection can be performed with excellent visualization of the spinal column and nerve roots. However, the combined approach increases the trauma for the patients, especially young patients (Peng, 2011).

A recent development has been the excision of hemivertebra by a posterioronly approach. Shono et al. (2001) reported successful results in correction of the deformities with the posterior-only excision of hemivertebrae in a series of 12 congenital kyphoscoliotic patients. Nakamura et al. (2002) reported the cases of five patients who underwent posterior-only excision of vertebrae with an average 12.8-year followup period. Ruf and Harms (2003), reported two series of congenital scoliosis that were treated by hemivertebra resection using a posterior-only approach.

Although the trauma on patients from the posterior-only approach is lower than that from the combined approach, the possible disadvantages of posterior-only procedures are that visualization may become difficult with blood loss; moreover, not all surgeons are technically comfortable with a posterior-only approach. Furthermore, spinal cord and dural manipulation increases the risk for neurologic injury.

Early surgery in very young children, however, requires a less invasive approach. In this series of children under 5 years of age, we excised hemivertebrae by a unilateral posterior approach and kept the concave side intact. The results show that no neurologic complications occurred. The average operative time was $142 \mathrm{~min}$, and the average blood loss was $114 \mathrm{ml}$. Compared with the bilateral posterior approach series reported in the literature the unilateral posterior approach used in our series had a shorter operative time and resulted in lower blood loss. Ruf and Harms (2003) used a posterior-only approach and bilateral exposure in very young children; the average operating time was $225 \mathrm{~min}$, and the average blood loss was $496 \mathrm{ml}$.

Hedequist and Emans (2004) reported 10 consecutive cases of congenital scoliosis that were treated with hemivertebra resection using a posterior-only approach, but with the three-rod technique. The average estimated blood loss was $270 \mathrm{ml}$. In our series, the results indicated that the unilateral posterior approach is a less traumatic, simple, and safe procedure.

For older children, pedicle screws have also been used. However, early correction in very young children also requires short and sufficiently rigid instrumentation. Therefore, some recent studies focused on transpedicular instrumentation. In 2003, Ruf and Harms first reported 25 cases of congenital scoliosis in very young children, with a mean age of 3 years and 4 months, who were operated on using bilateral transpedicular instrumentation for correction and fixation, and they achieved good results. The researchers stated that pedicle screws could be safely used even in children aged 1-2 years. However, Hedequist et al. have noted at their institution that the pedicles of young children, although adequate in size and shape for screw fixation, are less resistant to cephalad or caudal displacement than is required for initial correction and maintenance of correction after hemivertebra wedge resection. They used a third rod, with laminar fixation involving only those vertebrae already used for pedicle screw fixation to solve the problem. In our study, we only used unilateral transpedicular instrumentation for correction and fixationof scoliosis or kyphoscoliosis after hemivertebra resection. The results show 
that the correction rate of the main scoliosis curve was $65.9 \%$, and the correction rate of the segmental kyphotic angles was 78.1\%. These correction rates are almost in agreement with those reported by Ruf and Harms (2003).

Therefore, our results suggest that unilateral transpedicular instrumentation can provide sufficient force for correction and a short sufficiently rigid stabilization to maintain the correction of the deformity in children under 5 years of age when pedicle screws are successfully inserted. This technique allows for early mobilization without the necessity for wearing a cast for long periods. However, when the anatomy of adjacent pedicles is anomalous, it is difficult to insert the pedicle screw and/or achieve stable fixation. In that situation, adding laminar hooks and contralateral fixation might be a good option. For severe kyphotic patients, unilateral transpedicular instrumentation may not achieve and maintain satisfactory correction.

In addition to the advantages described above, unilateral transpedicular instrumentation has three potential advantages. First, leaving the concave side intact decreases the chance of injuring the spinal cord, as the spinal cord generally is shifted to the concave side. Second, the segmental curve may be corrected further over time because the concave growth is not affected. Compared with the period immediately after the operation, at the latest follow-up visit, three of our patients had achieved further correction of the segmental curve by over 21 . Third, a decrease in adverse effects is possible when only using unilateral transpedicular instrumentation; however, there are no adverse effects of pedicle screws on further growth. Although we have not measured the spinal canal diameters after surgery, no patient's neurologic function deteriorated over the follow-up period.
Posterior situ fusion is performed as convex fusion to avoid curve progression. Fusion in situ posterior using single rod and pedicle screw instrumentation. In congenital scoliosis is minimal blood loss, less traumatic, simple, safe and effective procedure. This study showed the early fusi in situ posterior of congenital scoliosis structural changes occur above or below can reduce fusion length, prevent curve progression and effectively achieve a more satisfactory correction without hazardous iatrogenic spinal stenosis, crankshaft phenomena, or neurological complications. Further study is needed to assess the progress of the outcome of surgery.

\section{AUTHOR CONTRIBUTION}

Pamudji Utomo, Adi Surya Dharma, nd Rieva Ermawan were contributed to do physical examination, run posterior situ fusion, and wrote the manuscript.

\section{CONFLICT OF INTEREST}

We declare that there was no conflict of interest.

\section{FUNDING AND SPONSORSHIP}

There was no external funding.

\section{ACKNOWLEDGEMENT}

We thank to Department of Orthopaedic \& Traumatology, Prof. Dr. R. Soeharso Orthopaedic Hospital, Surakarta, and parent of patients who give permission to conduct thi study.

\section{REFERENCE}

Bollini G, Docquier PL, Viehweger E, Launay F, Jouve JL (2006). Thoracolumbar hemivertebrae resection by double approach in a single procedure: longterm follow-up. Spine (Phila $\mathrm{Pa}$ 1976). 31(15): 1745-1757. https://doi.- 
Utomo et al./ Fusi in situ posterior for congenital scoliosis

org/10.1097/o1.brs.0000224176.404 57.52

Hedequist D, Emans J (2004). Congenital scoliosis. J Am Acad Orthop Surg. 12(4): 266-75. https://doi.org/10.5435/00124635-200407000-00007.

Nakamura H, Matsuda H, Konishi S, Yamano Y (2002). Single-stage excision of hemivertebrae via the posterior approach alone for congenital spine deformity: follow-up period longer than ten years. Spine (Phila Pa 1976). 27(1): 110-115. https://doi.org/10.1097/oo007632-200201010-00026

Peng X, Chen L, Zou X (2011). Hemivertebra resection and scoliosis correction by a unilateral posterior approach using single rod and pedicle screw instrumentation in children under 5 years of age. J Pediatr Orthop B. 20(6): 397-403. https://doi.org/10.1097/BPB.obo13e3283492060

Ruf M, Harms J (2003). Posterior hemivertebra resection with transpedicular instrumentation: early correction in children aged 1 to 6 years. Spine (Phila Pa 1976). 28(18): 2132-2138. https://doi.org/10.1097/01.BRS.ooo0084627.57308.4A

Shono Y, Abumi K, Kaneda K (2001). Onestage posterior hemivertebra resection and correction using segmental posterior instrumentation. Spine (Phila Pa 1976). 26(7): 752-757. https://doi.org/10.1097/00007632-200104010-00011. 